\title{
COMPOSITION OF BIOACTIVE SECONDARY METABOLITES AND MUTAGENICITY OF SAMBUCUS NIGRA L. FRUIT AT DIFFERENT STAGES OF RIPENESS
}

\author{
I. Koss-MikolajczyK*, A. Lewandowska, T. Pilipczuk, B. Kusznierewicz and A. Bartoszek \\ Department of Food Chemistry, Technology and Biotechnology, Gdańsk University of Technology, \\ 11/12 Narutowicza St., 80-233 Gdańsk. Poland
}

(Received: 17 July 2015; accepted: 26 November 2015)

\begin{abstract}
The relationship between the content of bioactive compounds and mutagenic activity of elderberry fruit at different stages of ripeness was investigated. Significant differences in the antioxidant profiles (TLC, HPLC with post-column derivatization) and antioxidant activity (ABTS, DPPH, and FC tests) were observed for studied elderberry extracts. The more ripen the fruit at the time of harvest were, the higher the content of anthocyanins (increase from 0 to 7.8 $\mathrm{mg} \mathrm{g}^{-1} \mathrm{~d}$.w.) and antioxidant activity of the extracts (about 5-fold increase) were. Cyanogenic glycosides were not detected at any stage of ripeness. Accordingly, Ames MPF test (Xenometrix) did not reveal any mutagenicity. Our study suggests that instability of cyanogenic glycosides ensures safety of food/pharmaceutical products based on even not fully ripen elderberry fruit.
\end{abstract}

Keywords: antioxidants, anthocyanins, cyanogenic glycosides, indolic auxins, mutagenicity

Elder (Sambucus nigra L.) is a widespread wild growing plant in Europe, Asia, North Africa, and North America. For thousands of years, S. nigra has been used in folk medicine as a cure for colds, asthma, arthritis, pain relief, inflammation, and even constipation. It is cultivated for ornamental purposes, but also it is a long recognised source of bioactive components. Because of consumers' growing interest in natural remedies, Sambucus nigra fruit and flowers in the form of standardized extracts are used as an alternative to synthetic drugs for treating influenza and other viral infections (ZAKAY-Rones et al., 2004; RosCHEK et al., 2009; KinOSHita et al., 2012; SwAminathan et al., 2013). However, elderberry based products can be potentially toxic, because bark, leaves, seeds were shown to contain cyanogenic glycosides, such as sambunigrin (Ulbricht et al., 2014). Regarding the fruit, unripe berries were suggested also to contain these substances, but to our best knowledge there are no specific data on the content of cyanogenic glycosides in S. nigra fruit.

The aim of this study was to investigate the relationship between the stage of ripeness of elderberry and composition of both health beneficial (phenolic compounds) as well as potentially toxic compounds (cyanogenic glycosides). To our knowledge, such a relationship has not been investigated so far. The following indices were determined: biologically active components by HPLC, total antioxidant activity by spectrophotometric methods (ABTS, $\mathrm{DPPH}$, and FC), antioxidant profiles by TLC, and post-column derivatization. To assess potential mutagenic effects of elderberry fruit extracts, the Ames MPF test was used.

\footnotetext{
* To whom correspondence should be addressed. Phone: +48 883524361; e-mail: iza.koss88@gmail.com
} 


\section{Materials and methods}

\subsection{Chemicals and reagents}

The following chemicals were used: 1,1-diphenyl-2-picrylhydrazyl (DPPH), 2,2-azinobis(ethyl-2,3-dihydrobenzothiazoline-6-sulphonic acid) diammonium salt (ABTS), FolinCiocalteu's phenol reagent (FC), HPLC grade acetonitrile and formic acid from Merck (Germany); standards: 6-hydroxy-2,5,7,8-tetramethylchroman-2-carboxylic acid (Trolox) and rutin from Sigma-Aldrich (USA), cyanidin-3-glucoside from Fluka (USA), and chlorogenic acid from Extrasynthese (France). HPLC grade solvents were from Chempur (Poland) or POCH (Poland). Water was purified with a QPLUS185 system from Millipore (USA).

\subsection{Plant material and extracts}

Elderberry fruit (about $0.5 \mathrm{~kg}$ ) at different stages of ripeness covering one growing season were hand harvested from the same shrub in a private garden in the area close to the University. The berries were randomly chosen from different parts of the shrub and kept at $-20{ }^{\circ} \mathrm{C}$ until studied. To obtain extracts, the fruit were freeze-dried using Christ Alpha 2-4 LSC, and $0.2 \mathrm{~g}$ of lyophilizate was suspended in $3 \mathrm{ml}$ of $70 \%$ methanol (chemical determinations) or deionized water (Ames MPF test) and placed in the ultrasound bath for $10 \mathrm{~min}$. Then, the suspensions were centrifuged (Heraeus Megafuge 16R Centrifuge) at 5000 r.p.m. for 20 min at $4{ }^{\circ} \mathrm{C}$ in order to remove particulates. The supernatants were used in further tests; for Ames MPF test they were additionally sterilized by passing through Millex sterile $\mathrm{R} 33 \mathrm{~mm}(0.22$ $\mu \mathrm{m})$ syringe-driven filters (Millipore).

To obtain methanolic macerates, $1 \mathrm{~g}$ portion of fresh fruit/leaves was mixed with $1 \mathrm{ml}$ ( $2 \mathrm{ml}$ in the case of leaves) of $70 \%$ methanol and macerated in a mortar. The macerates were centrifuged (Heraeus Megafuge 16R Centrifuge) at 5000 r.p.m. at $4{ }^{\circ} \mathrm{C}$ for $20 \mathrm{~min}$ in order to remove particulates. The supernatants were used for the determination of cyanogenic glycosides in fresh samples and after one day of storage at $4{ }^{\circ} \mathrm{C}$.

\subsection{Total antioxidant activity of fruit extracts by spectrophotometric methods}

The methods employing ABTS and DPPH radicals, as well as FC reagent, were used for the colorimetric determination of the total antioxidant activity as described earlier (KUSZNIEREWICZ et al., 2012).

\subsection{Determination of bioactive components by HPLC}

Quantitative determination of phenolic compounds in fruit extracts was performed using the Agilent 1200 Series HPLC-DAD-MS (High-performance liquid chromatography with photo diode array and mass spectrometer detectors) system (Agilent Technologies, USA) as described earlier (KuszNIEREwICZ et al., 2012). Chromatographic separations of cyanogenic compounds were performed on an Agilent Eclipse XDB-C8 column $(150 \times 4.6 \mathrm{~mm}, 3.5 \mu \mathrm{m})$. The conditions of chromatographic separation of cyanogenic glycosides present in elderberry extracts (70\% methanolic) were as follows: mobile phase: A: $0.1 \%(\mathrm{v} / \mathrm{v})$ formic acid in water, B: $0.1 \%(\mathrm{v} / \mathrm{v})$ formic acid in acetonitrile; elution program: $0 \mathrm{~min}: 5 \% \mathrm{~B}, 20 \mathrm{~min}: 30 \% \mathrm{~B}, 30$ min: $100 \%$, and $5 \mathrm{~min}$ post-run delay; flow rate: $1 \mathrm{ml} \mathrm{min}^{-1}$; injection volume: $6 \mu \mathrm{l}$. The mass detector was used in a scanning mode from 100 to 800 atomic mass units (amu). Drying gas 
temperature was $350{ }^{\circ} \mathrm{C}$ and nitrogen was the sheath gas. Data from the mass detector were collected, compiled, and analysed using Chemstation Rev.B.04.02.

The analysis of indolic auxins was performed by HPLC-DAD-FLD (High-performance liquid chromatography with photo diode array and fluorescence detectors) according to PilIPCZUK and co-workers (2015).

\subsection{Profiles of antioxidants using TLC and HPLC-coupled post-column derivatization}

Profiles of antioxidants in methanolic plant extracts studied were obtained either by TLC (Thin Layer Chromatography) using method described previously (KuszNIEREwicz et al., 2012) or by post-column online derivatization with ABTS reagent injected to eluate from HPLC column using Pinnacle PCX Derivatization Instrument (Pickering Laboratories, Inc., USA) according to KusZNIEREWICZ and co-workers (2011).

\subsection{Determination of mutagenicity by Ames MPF test}

The induction of mutations by elderberry extracts was studied using microplate version of Ames test (Ames MPF kit, Xenometrix, Switzerland). The procedure followed strictly producer's recommendations available from the website: http://www.xenometrix.ch/index. php?id=61.

\subsection{Statistical analysis}

All values were expressed as the mean $\pm \mathrm{SD}$ of three independent experiments unless stated otherwise. Correlations between antioxidant activity, anthocyanin content, and auxin content were examined using Pearson's correlation. Statistical differences among samples were estimated using one-way ANOVA (GraphPad Prism).

\section{Results and discussion}

The plant material for this study was collected over 10 weeks' period to obtain the whole spectrum of ripeness stages. Table 1 shows sample abbreviations and harvest date of elderberry fruit that were used throughout this study.

Table 1. Elderberry sample abbreviations and harvest date

\begin{tabular}{lll}
\hline Sample ID & Harvest date & Fruit colour \\
\hline D1 & 20.07 .2013 & green \\
D8 & 27.07 .2013 & green \\
D15 & 03.08 .2013 & green \\
D23 & 11.08 .2013 & green \\
D32 & 20.08 .2013 & red \\
D38 & 26.08 .2013 & mostly black \\
D46 & 03.09 .2013 & black \\
D53 & 10.09 .2013 & black \\
D60 & 17.09 .2013 & black \\
D64 & 21.09 .2013 & black \\
\hline
\end{tabular}




\subsection{TLC profiles of antioxidants}
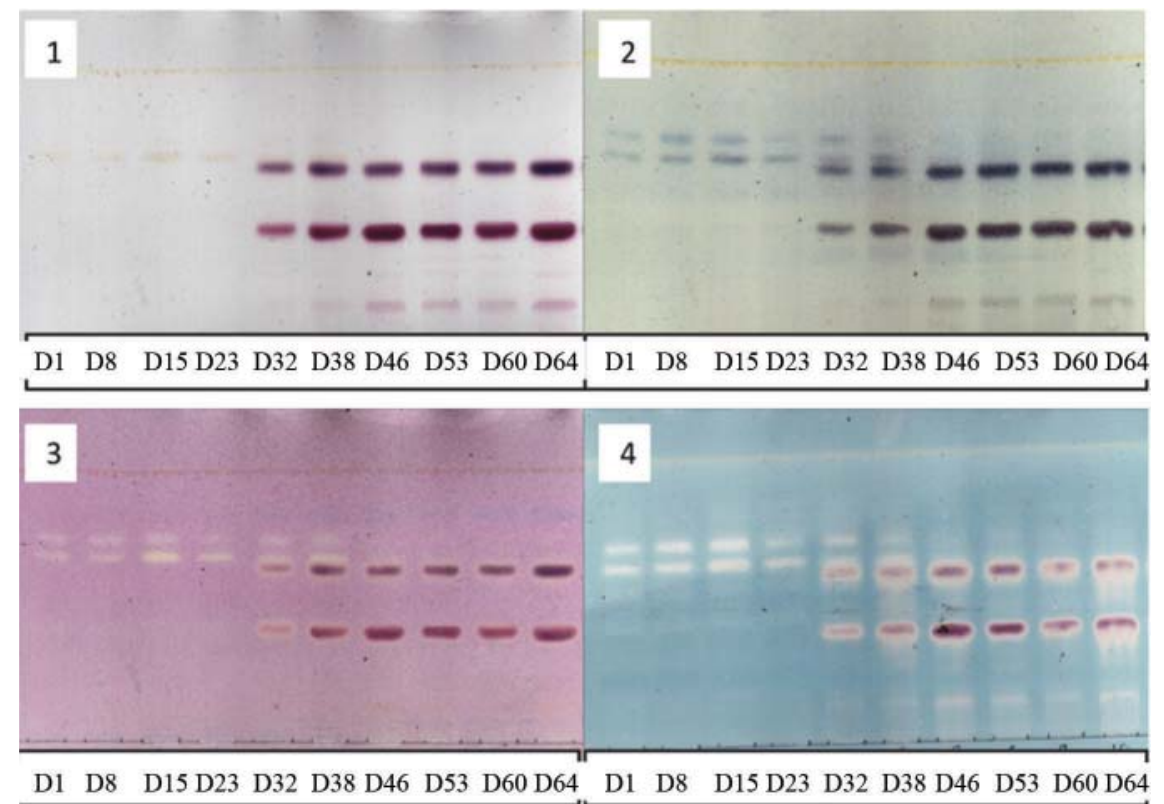

Fig. 1. Antioxidant profiles of extracts from Sambucus nigra fruit at different stages of ripeness obtained by TLC without (1) and with FC (2), DPPH (3), or ABTS (4) reagent visualization. Sample abbreviations (D1-D64) are as given in Table 1

TLC profiles of anthocyanins and other potential antioxidants present in extracts of elderberry fruit at different stages of ripeness are shown in Figure 1. Three major red pigments were detected from fruit harvested after 4 weeks of ripening. Their composition remained unchanged, only abundance seemed to increase with time. In contrast, antioxidant profiles of tested samples were changing significantly. For early stages of ripening, they were characterized by much poorer variety of antioxidative components than the ones obtained for ripe fruit. Moreover, the early occurring antioxidants possessed different chromatographic properties, hence different chemical structures from those occurring at later stages of ripeness. Two major redox active bands observed in unripe fruit gradually disappeared, while other constituents corresponding to anthocyanins begun to be formed.

\subsection{HPLC determination of bioactive compounds}

Phenols and indoles belong to two major families of phytochemicals with health-promoting properties documented in various biological models. The occurrence of these compounds may influence chemopreventive potential of elderberry fruit and products based on them. Figure 2 shows exemplary HPLC fingerprints of phenolic phytochemicals for three samples representative for consecutive stages of ripeness along with antioxidant profiles generated after derivatization with ABTS radical. The contents of phenolic phytochemicals identified are presented in Table 2. In unripe elderberry extracts, higher contents of neochlorogenic (Fig. 2, peak 1) and chlorogenic acid (Fig. 2, peak 2) than in the extracts from ripe fruit were 
observed. These two phenolic acids present in fruit at early stage of ripeness, appeared in TLC profiles as two fast migrating bands. Their contents, as well as the content of rutin (Fig. 2, peak 7) went down during fruit maturation.

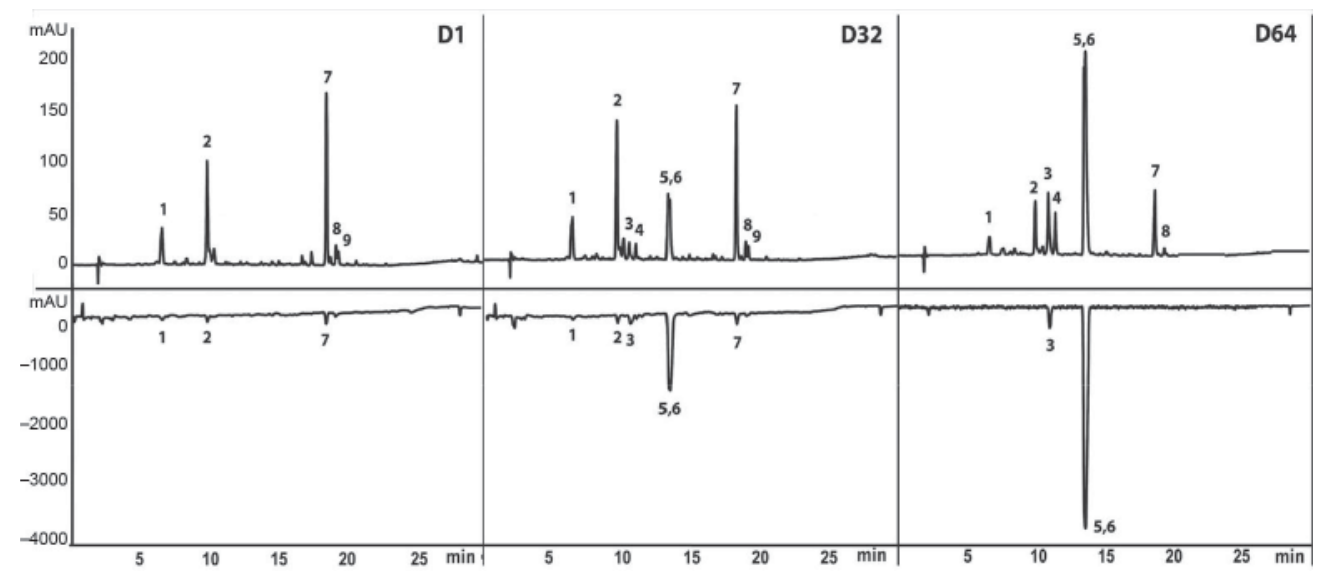

Fig. 2. HPLC profiles obtained for extracts of elderberry fruit at different stages of ripeness before and after derivatization with ABTS radical. The phenolic compounds were traced at $325 \mathrm{~nm}$ (top chromatograms), while bleaching of ABTS radical at $734 \mathrm{~nm}$ (bottom chromatograms). Numbered peaks represent: neochlorogenic acid (1), chlorogenic acid (2), cyanidin-3-O-sambubioside-5-O-glucoside (3), taxifolin glucoside (4), cyanidin-3-Osambubioside (5), cyanidin-3-O-glucoside (6), rutin (7), quercetin-3-O-6-acetylglucoside (8), Di-O-caffeoylquinic acid (9)

In accord with TLC profiles, in extracts of ripe elderberry, three main anthocyanins were identified. These were: cyanidin-3-O-sambubioside-5-O-glucoside identified by its $\left[\mathrm{M}^{+}\right]$at m/z 743 (Fig. 2, peak 3), cyanidin-3-O-sambubioside with $\left[\mathrm{M}^{+}\right]$at m/z 581 (Fig. 2, peak 5), and cyanidin-3-O-glucoside with $\left[\mathrm{M}^{+}\right]$at $\mathrm{m} / \mathrm{z} 449$ (Fig. 2, peak 6 ). These cyanidins are known to be the most common anthocyanins in elderberry fruit, which is confirmed by our results and those recently published by others (Youdim et al., 2000; LeE \& Finn, 2007; Duymuș et al., 2014; Mikulic-Petkovsek et al., 2014). The content of identified anthocyanins, once they appeared, increased rapidly with the stage of ripeness, but after about 6 weeks, the increases slowed down (Fig. 3). When fruit became fully ripen, the anthocyanin content remained more or less stable.

In contrast to anthocyanins, the contents of indolic compounds detected declined steadily with the stage of ripeness (Table 2). 
KOSS-MIKOŁAJCZYK et al.: METABOLITES AND MUTAGENICITY OF SAMBUCUS NIGRA L. 447

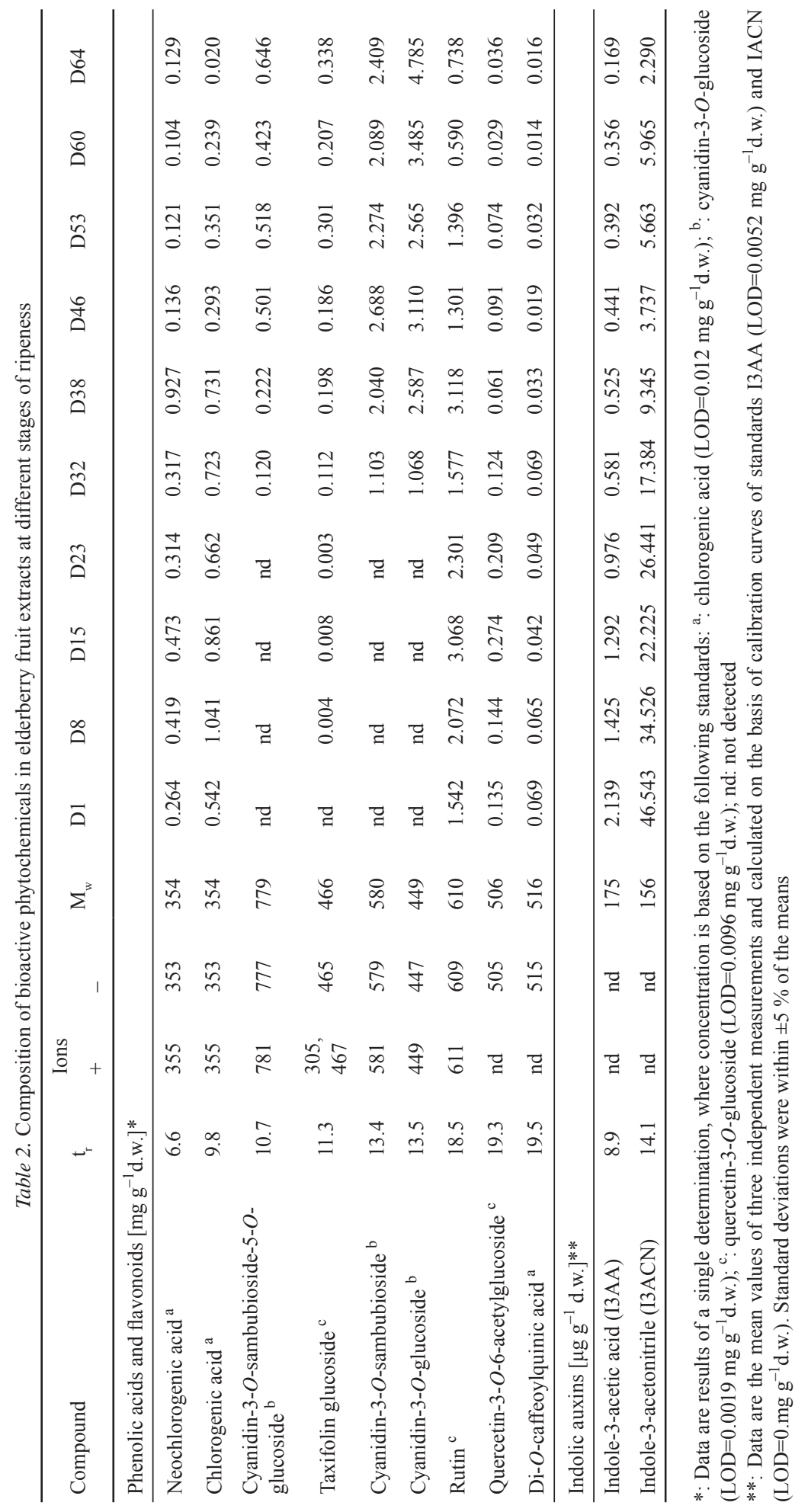




\subsection{Comparison of antioxidant activity}

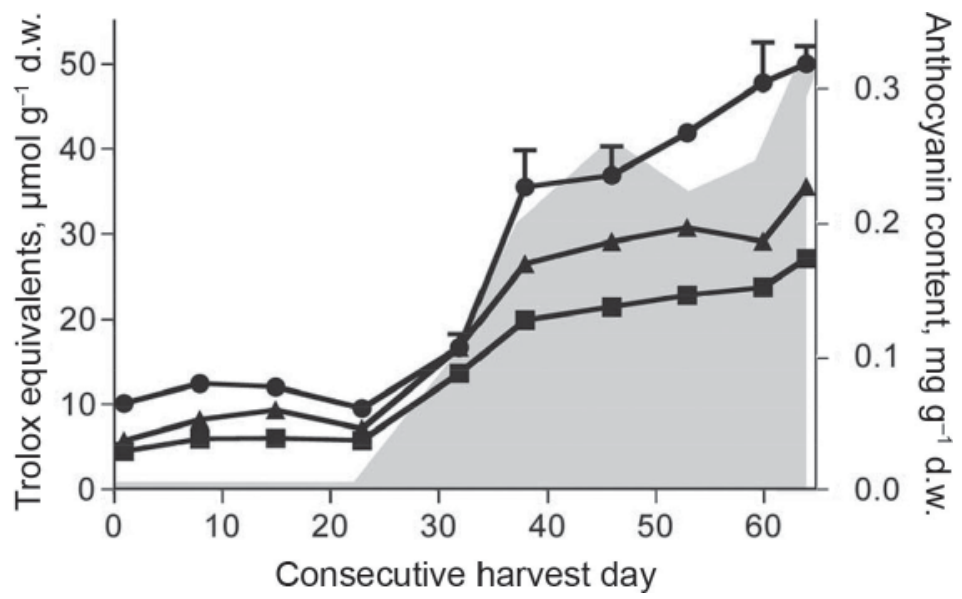

Fig. 3. Total antioxidant activity measured by ABTS (circles), DPPH (triangles), and FC (squares) tests and anthocyanin content determined by HPLC (grey area) for methanol extracts of elderberry fruit at different stages of ripeness. The results are means of three independent determinations (antioxidant activity) or a single determination (anthocyanins content)

Antioxidant capacities determined by standard spectrophotometric batch tests (ABTS, DPPH and FC) were highly correlated with the results obtained by HPLC with post-column derivatization. In general, the more ripen were the fruit, the higher the content of anthocyanins and antioxidant activity of these extracts were (Fig. 3). Significant increase in antioxidant activity of tested samples was visible since D32 (Table 3). The highest antioxidant activity among tested plant samples showed sample D64, which was collected as the last one in September. The lowest activity exhibited by the extracts D1 and D23 represented unripe fruit.

Table 3. Total antioxidant activity measured by ABTS, DPPH, and FC test for methanol extracts of elderberry fruit at different stages of ripeness

\begin{tabular}{lccc}
\hline Sample & $\begin{array}{c}\text { ABTS } \\
\left(\mu \mathrm{mol} \mathrm{g}^{-1} \text { d.w. }\right)\end{array}$ & $\begin{array}{c}\text { DPPH } \\
\left(\mu \mathrm{mol} \mathrm{g}^{-1} \text { d.w. }\right)\end{array}$ & $\begin{array}{c}\text { FC } \\
\left(\mu \mathrm{mol} \mathrm{g}^{-1} \mathrm{~d} . \mathrm{w} .\right)\end{array}$ \\
\hline D1 & $10.19 \pm 0.21$ & $5.79 \pm 0.21$ & $4.55 \pm 0.11$ \\
D8 & $12.53 \pm 0.20$ & $8.27 \pm 0.18$ & $6.01 \pm 0.12$ \\
D15 & $12.14 \pm 0.09$ & $9.43 \pm 0.05$ & $6.11 \pm 0.09$ \\
D23 & $9.59 \pm 0.09$ & $7.23 \pm 0.11$ & $5.85 \pm 0.16$ \\
D32 & $16.75 \pm 1.58^{*}$ & $16.80 \pm 0.23^{*}$ & $13.71 \pm 0.14^{*}$ \\
D38 & $35.57 \pm 4.32^{* *}$ & $26.58 \pm 0.49^{* *}$ & $19.95 \pm 0.56^{* *}$ \\
D46 & $36.98 \pm 3.39^{* *}$ & $29.19 \pm 0.43^{* *}$ & $21.53 \pm 0.14 * *$ \\
D53 & $41.96 \pm 0.80^{* *}$ & $30.81 \pm 0.34 * *$ & $22.92 \pm 0.23^{* *}$ \\
D60 & $47.84 \pm 4.68^{* *}$ & $29.24 \pm 0.37 * *$ & $23.79 \pm 0.09 * *$ \\
D64 & $50.08 \pm 2.01^{* *}$ & $35.64 \pm 0.36^{* *}$ & $27.20 \pm 0.18^{* *}$ \\
\hline
\end{tabular}

The results represent means \pm SD of three independent determinations. Significantly different values determined by one-way ANOVA with Dunnett's test were marked as: ${ }^{*} \mathrm{P}<0.05 ;{ }^{* *} \mathrm{P}<0.01$. 
Total antioxidant activity of the extracts numerically varied between batch tests, which is not surprising as they differ in substrate specificity, but the results were significantly correlated with each other as evidenced by the values above 0.97 of the Pearson coefficient (Table 4). Moreover, the comparison between the TE values determined by off-line tests (Fig. 3 ) and by post-column on-line derivatization (Fig. 2) revealed that the antioxidant activity of extracts studied came mainly from cyanidin-3-O-glucoside and cyanidin-3-O-sambubioside. The concentration of indoles was also strongly, albeit inversely, correlated with the antioxidant activity determined by FC, DPPH, or ABTS tests.

Table 4. Pearson coefficients for correlations between the content of major bioactive components of elderberry extracts and their antioxidant activity

\begin{tabular}{|c|c|c|c|c|c|c|}
\hline Pearson coefficient & I3ACN & $\begin{array}{c}\text { Anthocya- } \\
\text { nins }\end{array}$ & $\begin{array}{c}\text { Off-line } \\
\text { FC }\end{array}$ & $\begin{array}{c}\text { Off-line } \\
\text { DPPH }\end{array}$ & $\begin{array}{c}\text { Off-line } \\
\text { ABTS }\end{array}$ & $\begin{array}{c}\text { On-line } \\
\text { ABTS }\end{array}$ \\
\hline I3AA & 0.965 & -0.851 & -0.885 & -0.878 & -0.816 & -0.862 \\
\hline $\mathrm{I} 3 \mathrm{ACN}$ & & -0.895 & -0.916 & -0.924 & -0.869 & -0.890 \\
\hline Anthocyanins & & & 0.990 & 0.989 & 0.979 & 0.954 \\
\hline $\mathrm{FC}$ & & & & 0.996 & 0.979 & 0.979 \\
\hline DPPH & & & & & 0.976 & 0.979 \\
\hline Off-line ABTS & & & & & & 0.955 \\
\hline
\end{tabular}

\subsection{Assessment of risk}

HPLC-DAD-MS method used to determine the composition of cyanogenic glycosides in elderberry fruit extracts did not detect the presence of any components belonging to this group in tested samples. Because of such an unexpected result, it was decided to check whether this might be the effect of sample preparation or sample storage at low temperature, because it has been suggested before that this group of bioactive compounds is sensitive to low temperature. To test this possibility, the macerates were prepared in methanol from plant material expected to contain substantial amounts of cyanogenic glycosides, that is freshly harvested elder leaves and unripe, green fruit, as well as for comparison, semi-ripe (red fruit) and fully ripe (black fruit). The macerates were then analysed for the content of cyanogenic glycosides on the same day or after $24 \mathrm{~h}$ of storage at $4{ }^{\circ} \mathrm{C}$. In general, only trace amounts of components with appropriate $\mathrm{m} / \mathrm{z}$ value could be spotted in MS spectra. The highest signal for a peak corresponding to sambunigrin was detected in leaves, whereas the weak signal for this compound, still decreasing with the stage of ripeness, in elderberry fruit. Moreover, the signal for sambunigrin became undetectable after one day of storage of extracts in the fridge. All in all, the amounts of cyanogenic glycosides were too small to perform reliable quantification of their content based on HPLC analysis. It can be concluded, that the amount of components of elderberry fruit that could release toxic $\mathrm{HCN}$ at the level posing risk to human health can be rather excluded.

Another way of examining safety of the studied elderberry extracts relied on the assessment of their mutagenic activity performed by the microplate MPF version of Ames test. TA98 strain of Salmonella typhimurium, a standard strain for Ames test with broad spectrum of sensitivity, was used for this purpose. The observed mutagenicity induced by 
elderberry extracts, both with and without S9 fraction, was considerably lower than that for positive control - 2-aminoanthracene, for which the number of revertants reached the value of 42 for 48 being the maximum. Also, calculated according to the MPF Ames test producer's recommendations, fold induction in revertant number over the negative control was less than 2.0, which indicates no mutagenic effect. The mutagenicity of samples D46 to D60, which appeared to induce more revertants than other extracts, was not significantly higher than that of negative control representing spontaneous mutations (Fig. 4). Therefore, it can be concluded that the water extracts from elderberry fruit at different stages of ripeness show no mutagenic activity in S. typhimurium TA98 strain - neither without, nor with metabolic activation by rat liver $\mathrm{S} 9$ fraction.

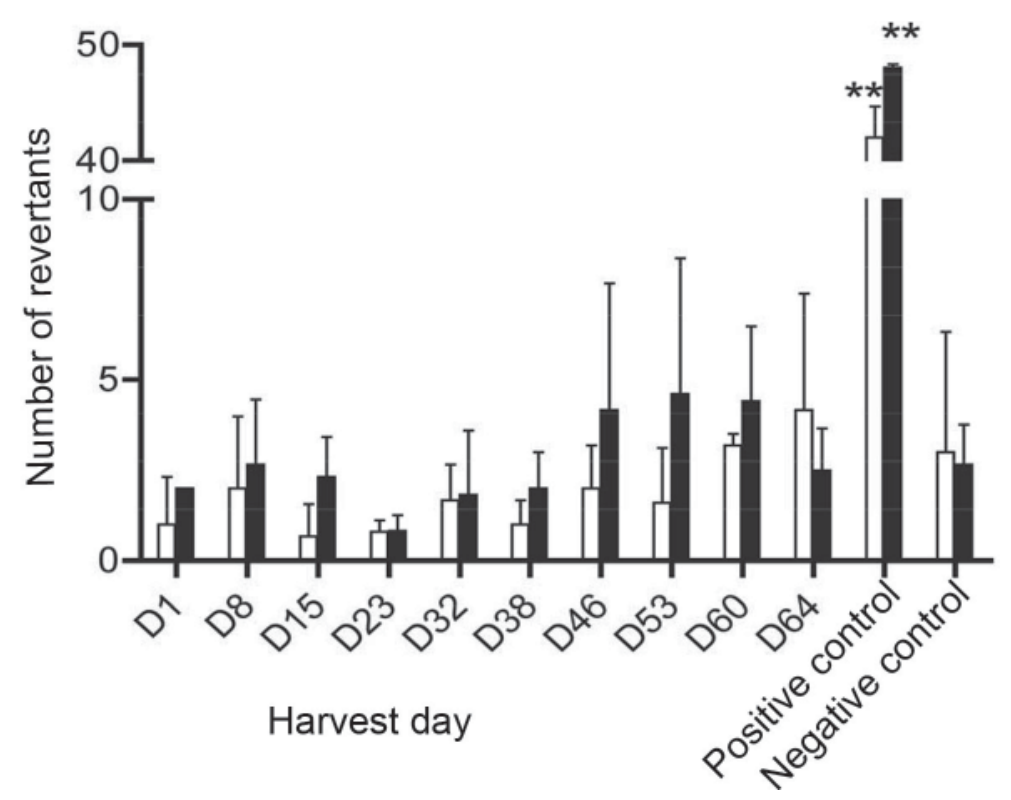

Fig. 4. Mutagenic activity of water extracts of elderberry fruit at different stages of ripeness determined by Ames MPF test in S. typhimurium strain TA98 with (black bars) and without (white bars) metabolic activation with S9 fraction. Positive controls were: 2-nitrofluorene (2NF, for TA98 -S9) and 2-aminoantracene (2-AA, for TA98 $+\mathrm{S} 9)$. The values are means \pm SD of 3 independent determinations. Significantly different values determined by one-way ANOVA with Dunnett's test were marked as: ${ }^{*} \mathrm{P}<0.05,{ }^{* *} \mathrm{P}<0.01$.

\section{Conclusions}

This study showed that the composition of bioactive components and antioxidant activity of extracts of elderberry differ substantially along the stages of ripeness. The antioxidant capacity, which is a potentially health promoting property of elderberry, increases. The redox active phytochemicals found in ripe fruit seem to interact antagonistically diminishing the antioxidant capacity of natural phytocomplex. The cyanogenic components of elderberry turned out to be either very unstable or occurred in ripe fruit in concentrations below LOD (Limit of the Detection). The studied extracts of S. nigra did not show any indication of mutagenic effect towards S. typhimurium TA98 strain at any stage of ripeness. Neither 
chemical composition nor biological tests used revealed any risk that could be associated with the consumption of elderberry based products, especially when they are prepared with fully ripen fruit.

We acknowledge the financial support of the Polish Ministry of Science and Higher Education (MNiSZW) in the form of research grant (N312 323039)

\section{References}

Duymuș, H.G., Göger, F. \& Bașer, K.H. (2014): In vitro antioxidant properties and anthocyanin composition of elderberry extracts. Food Chem., 155, 112-119.

Kinoshita, E., Hayashi, K., Katayama, H., Hayashi, T. \& Obata, A. (2012): Anti-influenza virus effects of elderberry juice and its fractions. Biosci. Biotech. Bioch., 76, 1633-1638.

Kusznierewicz, B., Piasek, A., Bartoszek, A. \& Namieśnik, J. (2011): Application of a commercially available derivatization instrument and commonly used reagents to HPLC on-line determination of antioxidants. $J$. Food Compos. Anal., 24, 1073-1080.

Kusznierewicz, B., Piekarska, A., Mrugalska, B., Konieczka, P., Namieśnik, J. \& Bartoszek, A. (2012): Phenolic composition and antioxidant properties of Polish blue-berried honeysuckle genotypes by HPLC-DAD-MS, HPLC postcolumn derivatization with ABTS or FC, and TLC with DPPH visualization. J. Agr. Food Chem., $60,1755-1763$.

LeE, J. \& FinN, C.E. (2007): Anthocyanins and other polyphenolics in American elderberry (Sambucus canadensis) and European elderberry (S. nigra) cultivars. J. Sci. Food Agr., 87, 2665-2675.

Mikulic-Petkovsek, M., Schmitzer, V., Slatnar, A., Todorovic, B., Veberic, R., Stampar, F. \& Ivancic, A. (2014): Investigation of anthocyanin profile of four elderberry species and interspecific hybrids. J. Agr. Food Chem., $62,5573-5580$.

PilipczuK, T., Dawidowska, N., Kusznierewicz, B., Namieśnik, J. \& Bartoszek, A. (2015): Simultaneous determination of indolic compounds in plant extracts by solid-phase extraction and high-performance liquid chromatography with UV and fluorescence detection. Food Anal. Method., 8, 2169-2177.

Roschek, B., Fink, R.C., McMichael, M.D., Li, D. \& Alberte, R.S. (2009): Elderberry flavonoids bind to and prevent H1N1 infection in vitro. Phytochemistry, 70, 1255-1271.

Swaminathan, K., Dyason, J.C., Maggion, A., Von Itzstein, M., \& Downard, K.M. (2013): Binding of a natural anthocyanin inhibitor to influenza neuraminidase by mass spectrometry. Anal. Bioanal. Chem., 405, 65636572 .

Ulbricht, C., Basch, E., Cheung, L., Goldberg, H., Hammerness, P., Isaac, R., Purkh, K., Khalsa, S., Romm, A., Rychlik, I., Varghesse, M., Weissner, W., Windsor, R.C. \& Wortley, J. (2014): An evidence-based systematic review of elderberry and elderflower (Sambucus nigra) by natural standard research collaboration. J. Diet. Suppl., 11, 80-120.

Youdim, K.A., Martin, A. \& JosEPh, J.A. (2000): Incorporation of the elderberry anthocyanins by endothelial cells increases protection against oxidative stress. Free Radical Bio. Med., 29, 51-60.

ZaKay-Rones, Z., Thom, E., Wollan, T. \& Wadstein, J. (2004): Randomized study of the efficacy and safety of oral elderberry extract in the treatment of influenza A and B virus infections. J. Int. Med. Res., 32, 132-140. 